Pacific Journal of Mathematics

FINITE-DIMENSIONAL PROPERTIES O 


\title{
FINITE-DIMENSIONAL PROPERTIES OF INFINITE-DIMENSIONAL SPACES
}

\author{
B. R. WENNER
Dedicated to Professor John H. Roberts on the occasion of his sixty-fifth birthday

A topological space is called locally finite-dimensional if every point has a neighborhood of finite (covering) dimensiondim. In the class of metric spaces, it is shown that every locally finite-dimensional space has small inductive dimension ind $\leqq \omega$, and is strongly countable-dimensional (hence is also countable-dimensional). Examples are given to shown that the converses of these statements are false, and that the property of being locally finite-dimensional neither implies nor is implied by that of having large inductive dimension Ind. Sum theorems are included, of which the following is representative: a metric space is strongly countable-dimensional iff it is the union of a locally countable collection of closed finite-dimensional, locally finite-dimensional, or strongly countable-dimensional subsets.

1. Introduction. Our purpose will be to examine relationships among certain classes of infinite-dimensional spaces in terms of finitedimensional subsets. All spaces will be metric, so the two dimension functions dim and Ind [cf. 6] may be used interchangeably in the finite case [4]. We shall also have occasion to mention the small inductive dimension ind [cf. 6], which in general does not coincide with the others [7].

Several classes of spaces which include infinite-dimensional spaces have recently been investigated. Each of the following properties determines such a class of spaces; the definitions have been stated in such a way as to emphasize the fact that each property is defined in terms of finite-dimensional subsets.

Definition 1. $X$ is countable-dimensional iff $X$ is the countable union of finite-dimensional subsets [5].

Definition 2. $X$ is strongly countable-dimensional ifi $X$ is the countable union of finite-dimensional closed subsets [5].

Definition 3. ind $X \leqq \omega$ iff for every $p \in X$ and every neighborhood $U$ of $p$, there exists a neighborhood $V$ such that $p \in V \subset U$ and ind $B(V)$ is finite [2].

DEFinition 4. Ind $X \leqq \omega$ iff for every closed set $F$ and open 
set $U$ with $F \subset U$, there exists an open set $V$ such that $F \subset V \subset U$ and Ind $B(V)$ is finite [9].

To these four properties we add a fifth which enjoys some interesting interrelationships with the above.

Definition 5. $X$ is locally finite-dimensional iff each point of $X$ has a finite-dimensional neighborhood.

Since every metric space is paracompact it follows from work of C. H. Dowker [1] that for any fixed integer $n, \operatorname{dim} X \leqq n$ if and only if each point of $X$ has a neighborhood $U$ with $\operatorname{dim} U \leqq n$. On the other hand, any locally finite union of finite-dimensional spaces $\left\{X_{\lambda}: \lambda \in \Lambda\right\}$ such that $\sup \left\{\operatorname{dim} X_{\lambda}: \lambda \in \Lambda\right\}=\infty$ (e.g., the space $Q$ in $\S 2)$ is an example of an infinite-dimensional, locally finite-dimensional space (see Lemma 1 below). It is immediate from the definition that every subspace of a locally finite-dimensional space is itself locally finite-dimensional, and that any finite product of locally finite-dimensional spaces is again locally finite-dimensional. Unions of locally finite-dimensional spaces will be discussed in $\S 3$.

The following Lemma will be a basic tool for the investigation of locally finite-dimensional spaces.

LEMma 1. $X$ is locally finite-dimensional iff $X$ has a locally finite (closed, open) cover of finite-dimensional subsets.

Proof. The necessity is an immediate consequence of the paracompactness of $X$. For the sufficiency we assume that $X$ has a locally finite cover $\mathscr{A}$ of finite-dimensional subsets; let $p \in X$, and $U$ be a neighborhood of $p$ which meets at most finitely many members, say $A_{1}, \cdots, A_{k}$, of $\mathscr{A}$. Then

$$
\operatorname{dim} U \leqq \operatorname{dim} \bigcup_{i=1}^{k} A_{i} \leqq \sum_{i=1}^{k} \operatorname{dim} A_{i}+(k-1)
$$

[6, Corollary to Theorem II. 4], so $U$ is a finite-dimensional neighborhood of $p$.

THEOREM 1. Every locally finite-dimensional space is strongly countable-dimensional (hence is countable-dimensional).

Proof. By Lemma 1 any locally finite-dimensional space $X$ has a locally finite closed cover $\mathscr{F}$ of finite-dimensional subsets. Then for each $n=1,2, \cdots$, the closed set $F_{n}=\cup\{F: F \in \mathscr{F}$ and $\operatorname{dim} F \leqq$ $n\}$ has dimension $\leqq n$ by the Sum Theoreom [6, Theorem II. 1], so 


$$
X=\bigcup_{n=1}^{\infty} F_{n}
$$

is strongly countable-dimensional by definition.

Theorem 2. If $X$ is locally finite-dimensional, then ind $X \leqq \omega$.

Proof. Let $p$ be a point of a locally finite-dimensional space $X$ and $U$ be a neighborhood of $p$. Then there exist neighborhoods $V$ and $W$ of $p$ such that $\operatorname{dim} V<\infty$ and $\bar{W} \subset V \cap U ; W$ is the desired neighborhood since

$$
\text { ind } B(W) \leqq \operatorname{dim} B(W) \leqq \operatorname{dim} V<\infty \text {. }
$$

2. Counterexamples. As a consequence of Theorems 1 and 2, we see that the class of locally finite-dimensional spaces is a subclass of the classes of spaces which satisfy respectively Definitions 1,2 , and 3. In this section we shall establish that these are the only possible inclusions which involve the class of locally finite-dimensional spaces. To this end we introduce the following examples.

Example 1. For all $n=1,2, \cdots$ we define the subset $Q_{n}$ of $E^{n}$ by the formula

$$
Q_{n}=\left\{\left(x_{1}, \cdots, x_{n}\right): 0 \leqq x_{i} \leqq 1 / n \text { for all } i=1, \cdots, n\right\}
$$

and

$$
Q=\bigcup_{n=1}^{\infty} Q_{n}
$$

For $x, y \in Q, x=\left(x_{1}, \cdots, x_{m}\right), y=\left(y_{1}, \cdots, y_{n}\right)$, we define

$$
\begin{array}{ll}
d(x, y)=\left(\sum_{i=1}^{n}\left(x_{i}-y_{i}\right)^{2}\right)^{1 / 2} & \text { if } m=n, \\
d(x, y)=1 & \text { if } m \neq n .
\end{array}
$$

It is easily verified that $d$ is a metric for $Q$.

Example 2. For all $n=1,2, \cdots$ we define the subset $J_{n}$ of the Hilbert cube by

$$
J_{n}=\left\{\left(x_{i}\right): 0 \leqq x_{i} \leqq 1 / n \text { for } i=1, \cdots, n \text {, and } x_{i}=0 \text { for } i>n\right\},
$$

and

$$
J=\bigcup_{n=1}^{\infty} J_{n}
$$


Note that $J$ is not homeomorphic to the well-known set $K_{\omega}$ which consists of all points of the Hilbert cube for which at most finitely many coordinates are nonzero.

Theorem 3. $Q$ is locally finite-dimensional, but $Q$ has no large transfinite dimension (i.e. Ind $Q$ does not exist).

Proof. If $p \in Q$ then $p \in Q_{n}$ for some $n$; $Q_{n}$ is then an $n$ dimensional neighborhood of $p$, which proves that $Q$ is locally finitedimensional. The remainder of the Theorem follows from a result of Ju. M. Smirnov [9, Remark, p. 193].

\section{THEOREM 4.}

(i) $J$ is strongly countable-dimensional (hence is also countabledimensional),

(ii) ind $J=\omega$,

(iii) Ind $J=\omega$, and

(iv) $J$ is not locally finite-dimensional.

Proof. That $J$ is strongly countable-dimensional is obvious; to prove the remaining assertions we shall need the following Lemmas.

Lemma 2. For any neighborhood $N$ of the origin $q$ of $J$, there exists an integer $k$ such that $J_{n} \subset N$ for all $n \geqq k$.

Proof. Any neighborhood $N$ of $q$ contains a sphere about $q$ of radius $(k-1)^{-1 / 2}$ for some suitably large $k$. If $n \geqq k$ and $x=\left(x_{i}\right) \in J_{n}$, then

$$
d(x, q)=\left(\sum_{i=1}^{\infty} x_{i}^{2}\right)^{1 / 2} \leqq\left(\sum_{i=1}^{n} 1 / n^{2}\right)^{1 / 2}=n^{-1 / 2}<(k-1)^{-1 / 2},
$$

so $x \in N$.

Lemma 3. The space $J-\{q\}$ is locally finite-dimensional.

Proof. For any $x \in J-\{q\}$ we let $V$ be a neighborhood of $x$ such that $q \notin \bar{V}$ (the closure of $V$ in $J$ ). Then $J-\bar{V}$ is a neighborhood of $q$, so by Lemma 2

$$
J-\bar{V} \supset \bigcup_{n=k}^{\infty} J_{n}
$$

for some integer $k$, which implies

$$
V \subset \bar{V} \subset J-\bigcup_{n=k}^{\infty} J_{n} \subset \bigcup_{n=1}^{k-1} J_{n}
$$


Thus $V$ is a subset of a finite-dimensional space and is itself finitedimensional.

Lemma 4. If $X$ is compact, then Ind $X \leqq \omega$ iff ind $X \leqq \omega$.

Proof. Only the sufficiency requires proof, so we suppose ind $X \leqq$ $\omega$ and let $F$ and $G$ be disjoint closed subsets of $X$. For each point $p \in F$ let $U(p)$ be a neighborhood of $p$ which misses $G$ and such that ind $B(U(p))=n(p)<\infty$ (hence Ind $B(U(p))=n(p)$ as $X$ is separable). As $F$ is compact there exist finitely many members of $F$, say $p_{1}, \cdots, p_{k}$, such that $F \subset \bigcup\left\{U\left(p_{i}\right): i=1, \cdots, k\right\}=U$. Clearly $U \cap G=\varnothing$, and

$$
B(U) \subset \bigcup_{i=1}^{k} B\left(U\left(p_{i}\right)\right)
$$

implies Ind $B(U) \leqq \max \left\{n\left(p_{i}\right): i=1, \cdots, k\right\}<\infty$.

We are now prepared to prove (ii) of Theorem 4. If $N$ is any neighborhood of $q$, then by Lemma 2 there exists an integer $k$ such that

$$
B(N) \subset J-N \subset J-\bigcup_{n=k}^{\infty} J_{n} \subset \bigcup_{n=1}^{k-1} J_{n},
$$

so $B(N)$ is finite-dimensional. On the other hand for any $x \in J-\{q\}$ and any neighborhood $U$ of $x$ there exists, by Lemma 3, a neighborhood $V$ whose closure has finite dimension. Then $U \cap V$ is a neighborhood of $x$ whose boundary is a subset of $\bar{V}$ and is therefore finite-dimensional. This implies ind $J \leqq \omega$, hence ind $J=\omega$ since $J$ contains subsets $J_{n}$ of arbitrarily high finite dimension $n$.

To prove (iii) it suffices, by Lemma 4 , to show $J$ is compact, so we let $A$ be an infinite subset of $J$. If $A$ is contained in $\bigcup_{n=1}^{k} J_{n}$ for some $k$ then $A$ has an accumulation point in that compact subset of $J$. If, on the other hand, there is no such $k$, then $q$ is an accumulation point of $A$, since for every neighborhood $N$ of $q$ there exists, by Lemma 2 , an integer $k$ such that

$$
N \cap A \supset\left(\bigcup_{n=k+1}^{\infty} J_{n}\right) \cap A \supset\left(J-\bigcup_{n=1}^{k} J_{n}\right) \cap A \neq \varnothing .
$$

Thus in all cases $A$ has an accumulation point and $J$ is compact.

Part (iv) follows from the fact that $q$ has no finite-dimensional neighborhood, since by Lemma 2 any neighborhood of $q$ contains sets of arbitrarily high dimension. This completes the proof of Theorem 4 .

In view of Theorem 3 and Theorem 4 we have now demonstrated the validity of the opening statement of this section. There is one other class of spaces which enjoys an interesting relationship with 
the class of locally finite-dimensional spaces:

DEFINITION 6. $X$ is weakly infinite-dimensional iff for every sequence of pairs $F_{i}, G_{i}$ of disjoint closed sets of $X$ there exist open sets $U_{i}, i=1,2, \cdots$, such that

$$
F_{i} \subset U_{i} \subset X-G_{i}
$$

and

$$
\bigcap_{i=1}^{k} B\left(U_{i}\right)=\varnothing \text { for some integer } k \text {. }
$$

This definition is due to Ju. M. Smirnov [11], and a space which satisfies this definition is sometimes called "weakly infinite-dimensional in the sense of Ju. M. Smirnov." As an immediate consequence of a remark of E. G. Sklyarenko [8, p. 201] we can state the following.

REMARK. No infinite-dimensional space is both locally finitedimensional and weakly infinite-dimensional.

3. The sum theorems. In this section we shall characterize several kinds of unions of finite-dimensional, locally finite-dimensional, and strongly countable-dimensional spaces; these may be compared with sum theorems for finite-dimensional and countable-dimensional spaces given in an earlier work [12]. We first give a sum theorem for locally finite-dimensional spaces.

THEOREM 5. $X$ is locally finite-dimensional iff $X$ is the union of a locally finite collection of closed locally finite-dimensional subsets.

Proof. Suppose $X=\bigcup\left\{F_{\lambda}: \lambda \in \Lambda\right\}$ where $F_{\lambda}$ is a closed locally finite-dimensional subset for each $\lambda \in \Lambda$. Then for each $\lambda \in \Lambda$ there exists, by Lemma 1 , a locally finite closed cover $\mathscr{F}_{\lambda}$ of $F_{\lambda}$ consisting of finite-dimensional subsets. We now show that the collection $\mathscr{F}=$ U $\left\{\mathscr{F}_{\lambda}: \lambda \in \Lambda\right\}$ is locally finite in $X$. For any $p \in X$ we let $M \subset \Lambda$ be the collection of $\mu \in \Lambda$ for which $p \in F_{\mu}$ ( $M$ is necessarily finite by hypothesis), and for each $\mu \in M$ we define the neighborhood $N(\mu)$ of $p$ by

$$
N(\mu)=X-\bigcup\left\{F: F \in \mathscr{F}_{\mu} \text { and } p \notin F\right\} .
$$

It is clear that

$$
N=\left(X-\bigcup_{\lambda \in \Lambda-M} F_{\lambda}\right) \cap\left(\bigcap_{\mu \in M} N(\mu)\right)
$$

is a neighborhood of $p$ which meets only finitely many members of 
F, since for each $\mu \in M$ the set $N(\mu)$ meets only finitely many members of ${ }^{\prime}$.

Hence $X$ has a locally finite cover $\mathscr{F}$ consisting of finitedimensional subsets, which implies that $X$ is locally finite-dimensional by Lemma 1.

The hypothesis of Theorem 5 cannot be significantly weakened. All subsets must be closed, as can be seen from the example $J=$ $(J-\{q\}) \cup\{q\}$ of a union of two locally finite-dimensional spaces (see Lemma 3) which is not itself locally finite-dimensional (by Theorem 4). On the other hard, the union must be taken over a locally finite collection since $J$ is the countable union of locally finite-dimensional spaces which is not locally finite-dimensional. More can be said about unions of locally finite-dimensional spaces, but we shall postpone further discussion until we have proven some infinite-dimensional analogues of the Sum Theorem [6, Section II. 2].

LEMma 5. $X$ is strongly countable-dimensional iff $X$ is the union of a locally finite collection of closed (open) strongly countabledimensional subsets.

Proof. By hypothesis $X$ has a closed cover $\left\{F_{\lambda}: \lambda \in \Lambda\right\}$ where $F_{\lambda}$ is strongly countable-dimensional for each $\lambda \in \Lambda$ (in the case of an open cover we shrink to a closed cover [3, p. 26] and proceed in exactly the same fashion), so for each $\lambda \in A$

$$
F_{\lambda}=\bigcup_{i=1}^{\infty} F_{\lambda, i}
$$

where each $F_{\lambda, i}$ is closed in $F_{\lambda}$ (hence in $X$ ) and is finite-dimensional. For each $i=1,2, \cdots$ we define

$$
G_{i}=\bigcup_{\lambda \in \Lambda} F_{\lambda, i}
$$

by Lemma 1, each $G_{i}$ is a closed, locally finite-dimensional subspace, hence is a closed, strongly countable-dimensional subspace by Theorem 1. This implies

$$
X=\bigcup_{i=1}^{\infty} G_{i}=\bigcup_{i=1}^{\infty}\left(\bigcup_{j=1}^{\infty} G_{i, j}\right)
$$

where each $G_{i, j}$ is a finite-dimensional closed subspace of $G_{i}$, hence of $X$, and the Lemma follows.

Theorem 6. $X$ is strongly conntable-dimensional iff $X$ is the union of a $\sigma$-locally finite collection of closed strongly countable- 
dimensional subsets.

Proof. Let $X=\bigcup_{i=1}^{\infty} F_{i}$ where each $F_{i}$ is the union of a locally finite collection of closed, strongly countable-dimensional subsets. Each $F_{i}$ is closed, and by Lemma 5 each $F_{i}$ is strongly countabledimensional, so

$$
X=\bigcup_{i=1}^{\infty}\left(\bigcup_{j=1}^{\infty} F_{i, j}\right)
$$

where for all $i, j=1,2, \cdots, F_{i j}$ is a closed, finite-dimensional subset of $F_{i}$ and hence of $X$, which completes the proof.

THEOREM 7. $X$ is strongly countable-dimensional iff each point of $X$ has a strongly countable-dimensional neighborhood.

Proof. If the condition is satisfied then $X$ has an open cover $\mathscr{U}$ all of the whose members are strongly countable-dimensional; then $\mathscr{U}$ has a locally finite open refinement, which proves the Theorem by Lemma 5 .

THEOREM 8. $X$ is strongly countable-dimensional iff $X$ is the union of a locally countable collection of closed strongly countabledimensional subsets.

Proof. By Theorem 7 it suffices to show that each $p \in X$ has a strongly countable-dimensional neighborhood. Let $U$ be a neighborhood of $p$ which meets at most countably many members $\left\{F_{i}: i=\right.$ $1,2, \cdots\}$ of the given collection; then

$$
U \subset \bigcup_{i=1}^{\infty} F_{i}=\bigcup_{i=1}^{\infty}\left(\bigcup_{j=1}^{\infty} F_{i, j}\right)
$$

where for all $i, j=1,2, \cdots, F_{i, j}$ is a finite-dimensional closed subset of $F_{i}$, hence of $X$, and the proof is complete.

THEOREM 9. $X$ is strongly countable-dimensional iff $S=\bigcup_{\lambda<\tau} F_{\lambda}$ where for all $\lambda<\tau, F_{\lambda}$ is strongly countable-dimensional and $\mathbf{U}_{\mu<\lambda} F_{\mu}$ is closed.

Proof. For all $i=1,2, \cdots$, all $\lambda<\tau$, we define

$$
F_{\lambda, i}=F_{\lambda}-S_{1 / i}\left(\bigcup_{\mu<\lambda} F_{\mu}\right)=\bigcup_{\mu \leq \lambda} F_{\mu}-S_{1 / i}\left(\bigcup_{\mu<\lambda} F_{\mu}\right) \text {. }
$$

By Theorem 6 it suffices to show that 


$$
\mathscr{F}=\bigcup_{i=1}^{\infty} \mathscr{F}_{i}=\bigcup_{i=1}^{\infty}\left\{F_{\lambda, i}: \lambda<\tau\right\}
$$

is a $\sigma$-locally finite closed cover of $X$. The second form of the definition of $F_{\lambda_{i}}$ shows that $\mathscr{F}$ is a closed collection; we now show that $\mathscr{F}$ covers $X$. If $p \in X$ then by hypothesis there exists a first $\lambda<\tau$ such that $p \in F_{\lambda}$. Also by hypothesis the set $\bigcup_{\mu<\lambda} F$ is closed, so there exists an integer $i$ such that

$$
d\left(p, \bigcup_{\mu<\lambda} F_{\mu}\right) \geqq 1 / i
$$

hence $p \in F_{\lambda, i}$.

Finally we show that each $\mathscr{F}_{i}$ is locally finite. If $p \in X$ there exists a first $\lambda<\tau$ such that $p \in F_{\lambda}$, and we shall prove that

$$
N=S_{1 / i}(p)-\bigcup_{\mu<\lambda} F_{\mu}
$$

is the desired neighborhood. For all $\mu<\lambda$,

$$
N \subset X-\bigcup_{\nu<\lambda} F_{\nu} \subset X-\bigcup_{\nu \leq \mu} F_{\nu} \subset X-F_{\mu, i} ;
$$

for all $\mu>\lambda$, on the other hand,

$$
F_{\mu, i} \subset X-S_{1 / i}\left(\bigcup_{\nu<\nu} F_{\nu}\right) \subset X-S_{1 / i}(p) \subset X-N .
$$

Thus $N \cap F_{\mu, i}=\varnothing$ unless $\mu=\lambda$, so $F_{i}$ is locally finite.

In view of Theorem 1 it is now possible to conclude our discussion of unions of locally finite-dimensional spaces with the following Corollaries of Theorems 6 , 8, and 9, respectively.

CoRollary 10. $X$ is strongly countable-dimensional iff $X$ is the union of a $\sigma$-locally finite collection of closed finite-dimensional (locally finite-dimensional) subsets.

CoRollary 11. $X$ is strongly countable-dimensional iff $X$ is the union of a locally countable collection of closed finite-dimensional (locally finite-dimensional) subsets.

CoRollaRy 12. $X$ is strongly countable-dimensional iff $X=$ $\mathrm{U}_{\lambda<\tau} F_{\lambda}$ where for all $\lambda<\tau, F_{\lambda}$ is finite-dimensional (locally finitedimensional) and $\bigcup_{\mu<\lambda} F_{\mu}$ is closed.

\section{REFERENCES}

1. C. H. Dowker, Local dimension of normal spaces, Quart. J. of Math., (Oxford) (2) 6 (1955), 101-120. 
2. W. Hurewicz and H. Wallman, Dimension Theory, Princeton, 1941.

3. S. Lefschetz, Algebraic Topology, Amer. Math. Soc. Colloquium, Vol. XXVII, 1942.

4. K. Morita, Normal families and dimension theory for metric spaces, Math. Ann., 128 (1954), 350-364.

5. J. Nagata, On the countable sum of zero-dimensional spaces, Fund. Math., 48 (1960), $1-14$.

6. - Modern Dimension Theory, Wiley, New York 1965.

7. P. Roy, Failure of equivalence of dimension concepts for metric spaces, Bull. Amer. Math. Soc., 68 (1962), 609-613.

8. E. Sklyarenko, On dimensional properties of infinite-dimensional spaces, Izv. Akad. Nauk. SSSR 23 (1959), 197-212; translated in Amer. Math. Soc. Transl., (2) 21 (1962), 35-50.

9. Ju. M. Smirnov, On universal spaces for certain classes of infinite-dimensional spaces, Izv. Akad. Nauk SSSR 23 (1959), 185-196; translated in Amer. Math. Soc. Transl., (2) 21 (1962), 21-33.

10. - On dimensional properties of infinite-dimensional spaces, Proc. I Prague Symp. (1961), 334-336.

11. - Some remarks on transfinite dimension, Sov. Math. Dokl., 2 (1961), 15721575 .

12. B. R. Wenner, Sums of finite-dimensional spaces, Bull. Australian Math. Soc., 1 (1969), 257-361.

Received April 19, 1971.

UNIVERSITY OF MISSOURI-KANSAS CITY 


\section{PACIFIC JOURNAL OF MATHEMATICS}

\section{EDITORS}

H. SAMELSON

Stanford University

Stanford, California 94305

C. R. HobBY

University of Washington

Seattle, Washington 98105
J. DUGUNDJI

Department of Mathematics

University of Southern California

Los Angeles, California 90007

RICHARD ARENS

University of California

Los Angeles, California 90024

\section{ASSOCIATE EDITORS}

E. F. BECKENBACH

B. H. NeumanN

F WoLF

K. YoshidA

\section{SUPPORTING INSTITUTIONS}

UNIVERSITY OF BRITISH COLUMBIA

UNIVERSITY OF SOUTHERN CALIFORNIA

CALIFORNIA INSTITUTE OF TECHNOLOGY

UNIVERSITY OF CALIFORNIA

MONTANA STATE UNIVERSITY

STANFORD UNIVERSITY

UNIVERSITY OF NEVADA

NEW MEXICO STATE UNIVERSITY

OREGON STATE UNIVERSITY

UNIVERSITY OF OREGON

OSAKA UNIVERSITY

UNIVERSITY OF TOKYO

UNIVERSITY OF UTAH

WASHINGTON STATE UNIVERSITY

UNIVERSITY OF WASHINGTON

$\stackrel{*}{*} \stackrel{*}{*} \stackrel{*}{*}{ }^{*}{ }^{*}$ MMEICAN MATHEMATICAL SOCIETY

NAVAL WEAPONS CENTER

Printed in Japan by International Academic Printing Co., Ltd., Tokyo, Japan 


\section{Pacific Journal of Mathematics}

\section{Vol. 42, No. $1 \quad$ January, 1972}

Tage Bai Andersen, On Banach space valued extensions from split faces ........

David Marion Arnold, A duality for quotient divisible abelian groups of finite

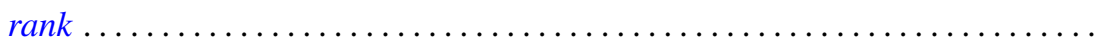

Donald Pollard Ballou, Shock sets for first order nonlinear hyperbolic

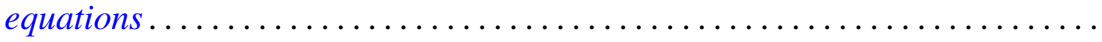

Leon Brown and Lowell J. Hansen, On the range sets of $H^{p}$ functions .........

Alexander Munro Davie and Arne Stray, Interpolation sets for analytic

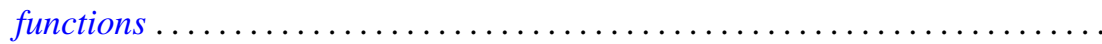

M. G. Deshpande, Structure of right subdirectly irreducible rings. II . . . . . . . . .

Barry J. Gardner, Some closure properties for torsion classes of abelian

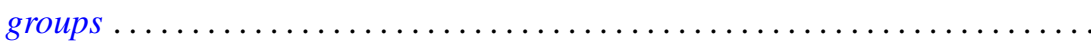

Paul Daniel Hill, Primary groups whose subgroups of smaller cardinality are

direct sums of cyclic groups . . . . . . . . . . . . . . . . . . .

Richard Allan Holzsager, When certain natural maps are equivalences .........

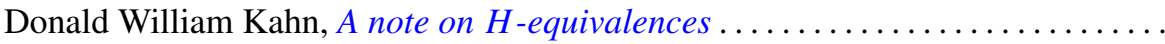

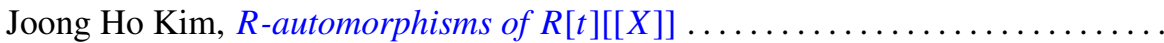

Shin'ichi Kinoshita, On elementary ideals of polyhedra in the 3-sphere.........

Andrew T. Kitchen, Watts cohomology and separability...

Vadim Komkov, A technique for the detection of oscillation of second order

ordinary differential equations .

Charles Philip Lanski and Susan Montgomery, Lie structure of prime rings of characteristic 2

Andrew Lenard, Some remarks on large Toeplitz determinants . .

Kathleen B. Levitz, A characterization of general Z.P.I.-rings. II .

Donald A. Lutz, On the reduction of rank of linear differential systems

David G. Mead, Determinantal ideals, identities, and the Wronskian ...

Arunava Mukherjea, A remark on Tonelli's theorem on integration in product

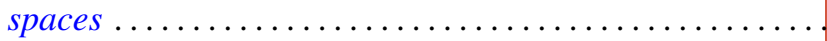

Hyo Chul Myung, A generalization of the prime radical in nonassociative rings.

John Piepenbrink, Rellich densities and an application to unconditionally nonoscillatory elliptic equations.

Michael J. Powers, Lefschetz fixed point theorems for a new class of multi-valued maps .

Aribindi Satyanarayan Rao, On the absolute matrix summability of a Fourier

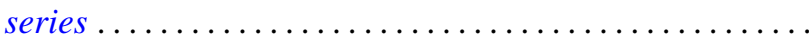

T. S. Ravisankar, On Malcev algebras ......................... 227

William Henry Ruckle, Topologies on sequences spaces . . . . . . . . . . . . . 235

Robert C. Shock, Polynomial rings over finite dimensional rings . . . . . . . . . 251

Richard Tangeman, Strong heredity in radical classes . . . . . . . . . . . . . . 259

B. R. Wenner, Finite-dimensional properties of infinite-dimensional spaces . . . . 267 